\title{
Rotational state-changing cold collisions of hydroxyl ions with helium
}

\author{
Daniel Hauser, Seunghyun Lee, Fabio Carelli, Steffen Spieler, Olga Lakhmanskaya, Eric S. Endres, \\ Sunil S. Kumar, Franco Gianturco and Roland Wester*
}

Cold molecules are important for many applications ${ }^{1}$, from fundamental precision measurements ${ }^{2}$, quantum information processing ${ }^{3}$, quantum-controlled chemistry ${ }^{4}$, to understanding the cold interstellar medium ${ }^{5}$. Molecular ions are known to be cooled efficiently in sympathetic collisions with cold atoms or ions ${ }^{6-8}$. However, little knowledge is available on the elementary cooling steps, because the determination of quantum state-to-state collision rates at low temperature is very challenging for both experiment and theory. Here we present a method to manipulate molecular quantum states by non-resonant photodetachment. Based on this we provide absolute quantum scattering rate coefficients under full quantum state control for the rotationally inelastic collision of hydroxyl anions with helium. Experiment and quantum scattering theory show reasonable agreement without adjustable parameters. Very similar rate coefficients are obtained for two different isotopes, which is linked to several quantum scattering resonances appearing at different energies. The presented method is also applicable to polyatomic systems and will help elucidate non-radiative processes in polyaromatic hydrocarbons and protein chromophores.

Molecular collisions at low temperatures have become known as a feature-rich quantum mechanical scattering problem. Theoretical calculations more than a decade ago have shown that a plethora of scattering resonances become accessible once the thermal de Broglie wavelength of the collision system becomes comparable to the effective range of the intermolecular interactions ${ }^{9}$. Understanding the quantum dynamics of cold molecular interactions has created a rapidly growing field during the past decade $\mathrm{e}^{10-12}$. Here, state-to-state inelastic collisions are among the most fundamental processesand also the most difficult to study. Cold inelastic collisions of neutral $\mathrm{OH}$ molecules interacting with rare gas atoms ${ }^{13}$ and, more recently, NO radicals ${ }^{14}$ can be explored using the Stark decelerator technique. In rotationally inelastic collisions of $\mathrm{O}_{2}$ and $\mathrm{H}_{2}$, scattering resonances due to specific partial waves could be observed ${ }^{15}$. Cold chemical reactions of neutral molecules have been studied and the significance of isotope effects in quantum shape resonances ${ }^{16}$ and the importance of tunnelling dynamics ${ }^{17}$ have been identified. For molecular ions several cooling schemes have been developed for vibrational and rotational cooling ${ }^{6,8,18-20}$. Initial state-dependent collisions have previously been studied at higher temperature using laser-induced reactions ${ }^{21,22}$, whereas rotational-state-resolved reactive collisions have recently been achieved at temperatures below $20 \mathrm{~K}$ (refs 7,23).

Here, we present a versatile scheme to manipulate the rotational quantum states of negative ions-the state-selective removal of ions by threshold photodetachment-to study the fundamental inelastic process of rotational-state-changing collisions. With this approach

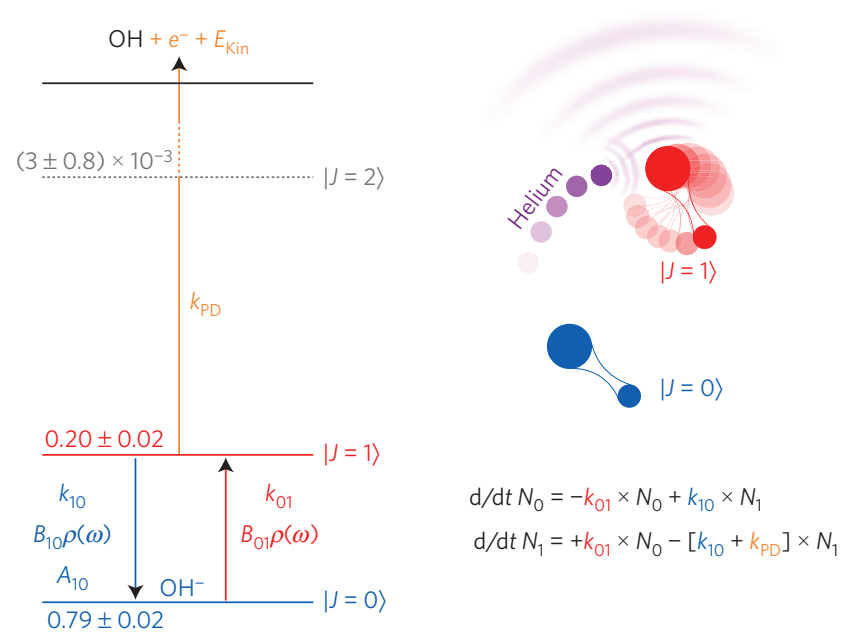

Figure 1 | $\mathrm{OH}^{-}$rotational energy levels and coupling rates. Diagram of the rotational energy levels of $\mathrm{OH}^{-}$, which are coupled by collisions with helium (with inelastic rate coefficients $k_{10}$ and $k_{01}$, see the schematic view on the right) and blackbody-radiation-induced transitions (with the Einstein coefficients $A_{10}, B_{10}$ and $B_{01}$, and radiation density $\rho(\omega)$ ). Photodetachment transitions with the rate $k_{P D}$ couple the excited anion states to the rotational ground state of neutral $\mathrm{OH}$ and a free electron. Given the spectroscopically measured populations of the rotational levels, shown next to each energy level, transitions involving $J=2$ can safely be neglected. The number of ions in $J=0$ and $J=1$ follows the presented rate equation model, where radiation-induced transitions are not included as they are too small to be effective (see Supplementary Information for details).

we determine the collision rate coefficients for the transition from the first excited $J=1$ state to the $J=0$ ground state of the hydroxyl negative ion and its deuterated isotopologue (see also Fig. 1)

$$
\begin{gathered}
{ }^{16} \mathrm{OH}^{-}(J=1)+{ }^{4} \mathrm{He} \rightarrow{ }^{16} \mathrm{OH}^{-}(J=0)+{ }^{4} \mathrm{He} \\
{ }^{16} \mathrm{OD}^{-}(J=1)+{ }^{4} \mathrm{He} \rightarrow{ }^{16} \mathrm{OD}^{-}(J=0)+{ }^{4} \mathrm{He}
\end{gathered}
$$

at a temperature of $22 \mathrm{~K}$. This is the anion rotational temperature, determined using photodetachment spectroscopy, as described in the Supplementary Information. Its value is also a good approximation for the effective temperature of the relative translational motion between the hydroxyl anions and the helium atoms. Simulations have verified that radiative excitation of the ions due to blackbody radiation does not need to be considered. 
a

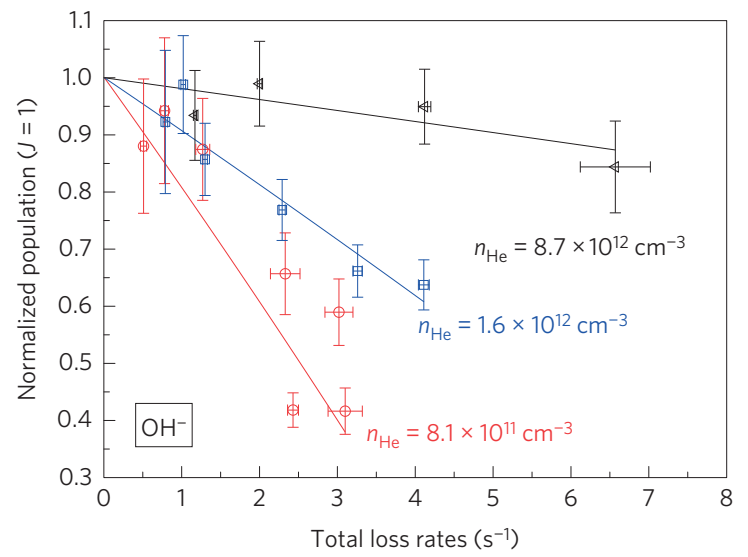

b

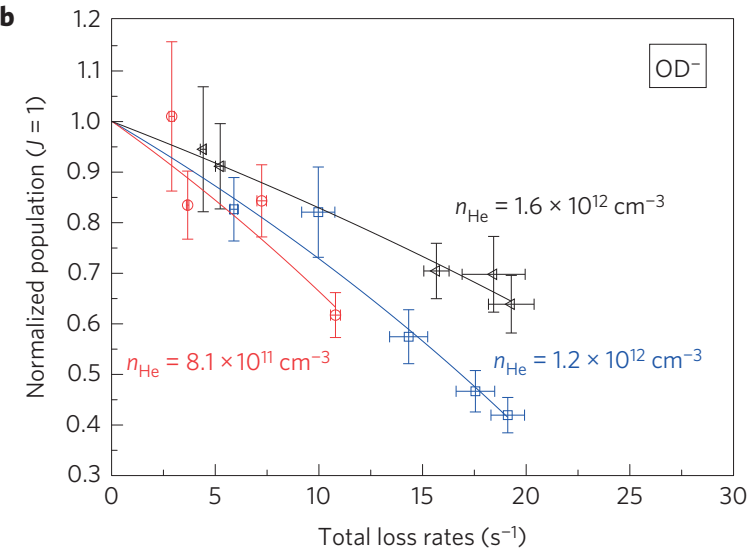

C

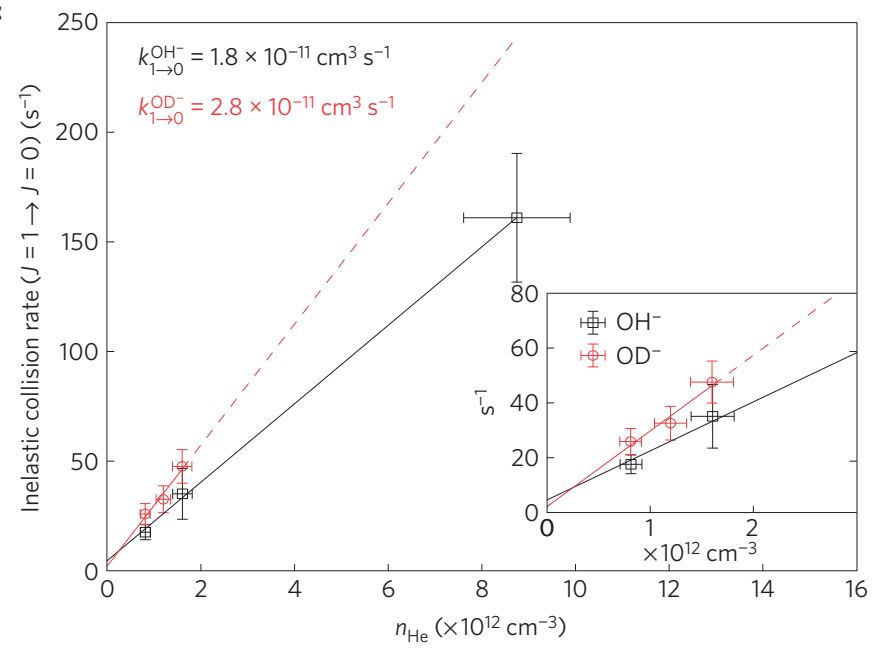

Figure 2 | Measured inelastic rate coefficients. $\mathbf{a}, \mathbf{b}$, Plot of the relative populations of $\mathrm{OH}^{-}$(a) and $\mathrm{OD}^{-}$(b) ions in the $J=1$ rotational state versus the

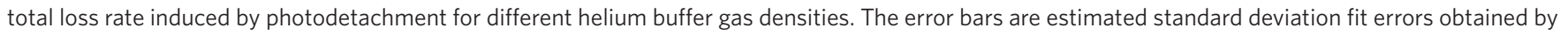
fitting exponential decay rates to the ion intensity as a function of storage time. The solid lines show fits to the solution of the rate equation system based on the parameters shown in Fig. 1 with the inelastic rate $k_{10}$ as the only free parameter. $\mathbf{c}$, Inelastic rates, as obtained from the fits to panels $\mathbf{a}$ and $\mathbf{b}$ as a

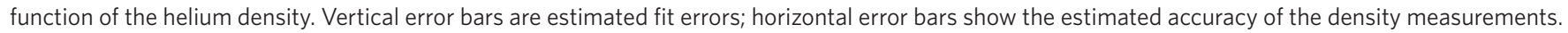
The slopes of the linear fits to these data points represent the inelastic collision rate coefficients for $\mathrm{OH}^{-}$and $\mathrm{OD}^{-}$. The inset shows a zoom of the data points at lower density.

The hydroxyl ions are perfect representations of a quantum mechanical rigid rotor, as neither electronic nor nuclear spin momentum couples appreciably to the mechanical rotation of the molecule. At long range the interaction potential does not distinguish between cations and anions, but at close proximity substantially different interaction energies are found as a function of distance and angle of approach. This influences reactive scattering of negative ions ${ }^{24}$ and is also crucial for the studied inelastic collisions. For the $\mathrm{OH}^{-}-\mathrm{He}$ system it leads to a much weaker interaction at short range than for similar sized cations, and suppresses the formation of bound $\mathrm{OH}^{-}-\mathrm{He}$ complexes even at $4 \mathrm{~K}$ (ref. 25). The interactions of the two isotopes $\mathrm{OH}^{-}$and $\mathrm{OD}^{-}$are described by the same anisotropic interaction potential ${ }^{26}$, with the effect that different collision rates directly refer to differences in the nuclear quantum dynamics.

To selectively probe only the upper rotational state of the ion, an intense photodetachment laser beam depletes the excited $J=1$ state, but leaves the ground state anions intact. This laser drives the detachment reaction

$$
\begin{aligned}
& { }^{16} \mathrm{OH}^{-}(J=1)+h v \rightarrow{ }^{16} \mathrm{OH}(J=3 / 2)+e^{-} \\
& { }^{16} \mathrm{OD}^{-}(J=1)+h v \rightarrow{ }^{16} \mathrm{OD}(J=3 / 2)+e^{-}
\end{aligned}
$$

The effect of the two reactions (1) and (2) on an ensemble of cold trapped ions is described by a simple system of rate equations, as depicted in Fig. 1 and described in the Supplementary Information. In the weak intensity limit the detachment laser induces a loss rate for the molecular ions that is proportional to the ion fraction in the $J=1$ state and to the laser intensity ${ }^{27,28}$. For strong enough laser intensities the rate for photodetachment becomes comparable to the inelastic collision rate and induces a depletion of the excited state. In the experiment this leads to a deviation from the linear intensity dependence of the induced loss rate. Once the detachment rate is much larger than the inelastic collision rate, only the latter determines the loss rate, because all molecular ions that reach the excited rotational state are immediately neutralized. In this discussion the effect of the second excited rotational state has been neglected, which is a safe assumption at the low temperatures in the ion trap (see Fig. 1 and Supplementary Information). The degenerate magnetic sub-levels of the excited rotational states have not been treated separately, as they will mix rapidly by collisions and interactions with the trapping potential.

The measurements were performed on ions trapped inside a cryogenic multipole radiofrequency ion trap ${ }^{6}$. The experimental set-up and the procedure of ion preparation and loading have been presented previously ${ }^{28}$, specific details are described in the 


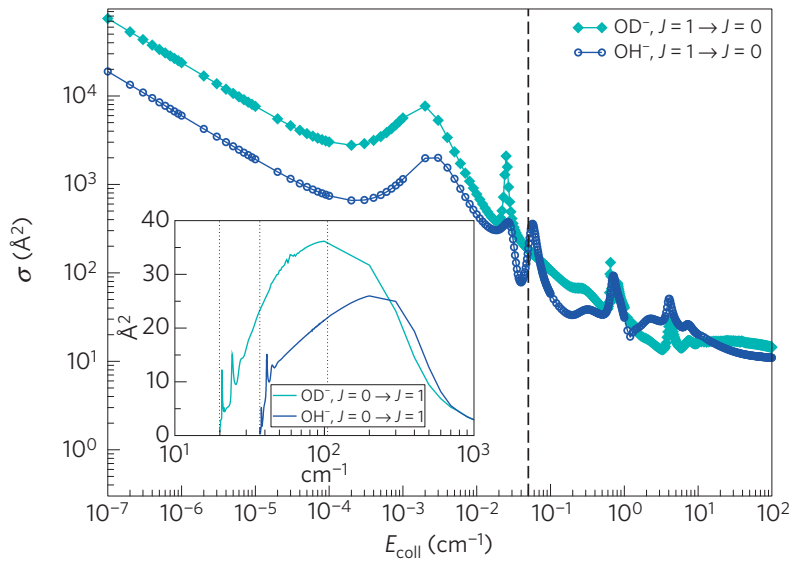

b

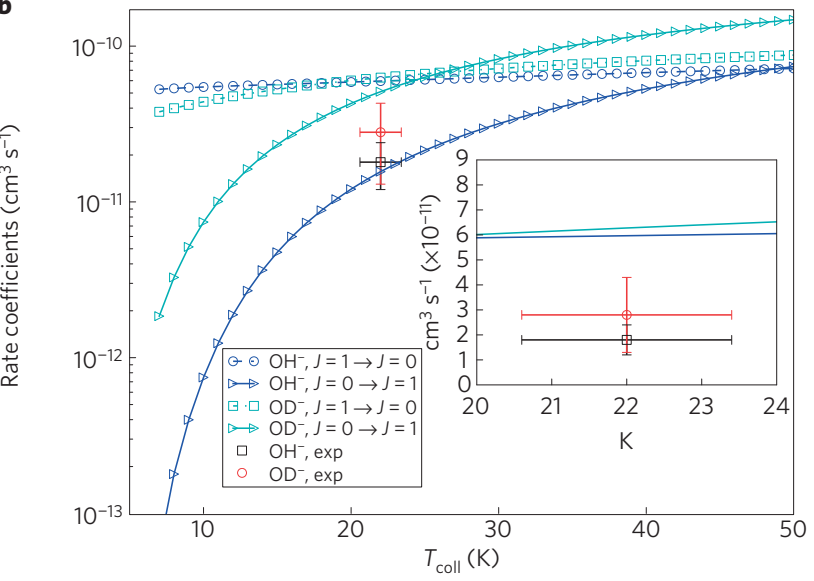

Figure 3 | Calculated inelastic cross-sections and rate coefficients. a, Inelastic scattering cross-section for $\mathrm{OH}^{-}$and $\mathrm{OD}^{-}$transitions from $J=1$ to 0 , calculated as a function of collision energy. Inset: inelastic excitation cross-sections from $J=0$ to 1 . $\mathbf{b}$, Temperature-averaged inelastic rate coefficients for excitation and de-excitation as a function of translational temperature. The dashed line in the upper panel marks the lowest relative energy that contributes significantly to the convolution of the thermal rate coefficients. Also shown are the measured rate coefficients with their respective error bars (see inset for a zoom with a linear axis scale).

Supplementary Information. Once loaded into the trap, a few hundred hydroxyl anions are subjected to the photodetachment laser beam, the frequency of which is tuned $5-20 \mathrm{~cm}^{-1}$ below the electron affinity of $\mathrm{OH}(\mathrm{OD})$ (see Fig. 1). This neutralizes only $\mathrm{OH}^{-}$ $\left(\mathrm{OD}^{-}\right)$anions in $J=1$ (and above) and forms a rotational ground state $\mathrm{OH}(\mathrm{OD})$ with total angular momentum $J=3 / 2$.

The exponential decay rates of trapped hydroxyl anions are determined from the stored ion signal as a function of the storage time in the trap for increasing intensity of the photodetachment laser. These rates are directly proportional to the population in the $J=1$ state. Plotting the ions' relative population as a function of their loss rate shows that with increasing loss rate, caused by increased photodetachment laser intensity, the excited state population is depleted. This is shown in Fig. 2a,b for $\mathrm{OH}^{-}$and $\mathrm{OD}^{-}$for different helium densities in the ion trap. At the highest helium density the normalized population remains almost constant and thereby unaffected by the depletion action of the detachment laser, because the detachment rate remains weak in comparison with the inelastic collision rates. However, at lower helium densities a depletion of the excited $J=1$ state by more than $50 \%$ is observed. By fitting the rate equation model shown in Fig. 1 to the data, the inelastic collision rates are extracted as a function of helium density. These are shown as a function of density in
Fig. 2c. The slope of the linear dependence yields the collision rate coefficients. They turn out to be almost the same for $\mathrm{OH}^{-}$ and $\mathrm{OD}^{-}$, amounting to $\left(1.8 \pm 0.4_{\text {stat }} \pm 0.2_{\text {syst }}\right) \times 10^{-11} \mathrm{~cm}^{3} \mathrm{~s}^{-1}$ and $\left(2.8 \pm 1.1_{\text {stat }} \pm 0.4_{\text {syst }}\right) \times 10^{-11} \mathrm{~cm}^{3} \mathrm{~s}^{-1}$, respectively. The statistical accuracy of the two values is caused by the precision of the exponential decay rate measurements and the temperature determination by photodetachment spectroscopy, which is also traced back to decay rate measurements. The systematic accuracy stems from the absolute helium density calibration. The result for the ratio of the two rates yields $k_{\mathrm{OH}^{-}} / k_{\mathrm{OD}^{-}}=0.6 \pm 0.3$; here, the accuracy of the absolute density calibration cancels.

Both collision rate coefficients are smaller than the Langevin capture rate coefficient, which assumes unit scattering probability for all partial waves up to the maximum quantum number that allows the collision partners to overcome the centrifugal barrier. This Langevin rate is $5.8 \times 10^{-10} \mathrm{~cm}^{3} \mathrm{~s}^{-1}$ for both $\mathrm{OH}^{-}$and $\mathrm{OD}^{-}$colliding with helium. Normalizing the measured rates to the Langevin model, we find the inelastic collision probability for $\mathrm{OH}^{-}$and $\mathrm{OD}^{-}$to be about $4 \%$. To understand why both isotopologues feature a similar, finite probability in inelastic collisions with helium, we have performed $a b$ initio quantum scattering calculations for both anions on the same interaction potential surface ${ }^{26}$ (neglecting non-Born-Oppenheimer effects) using the time-independent quantum coupled-channel method ${ }^{29}$. In the framework of the rigid-rotor approximation the ground vibrational state of the hydroxyl anion and the first ten asymptotic rotational states were taken into consideration. Partial waves up to an angular momentum of $40 \hbar$ were considered.

The calculations yield the collision energy-dependent integral scattering cross-sections, from which the thermal rate coefficients are obtained by proper averaging (shown in Fig. 3a,b). The ratio of excitation and de-excitation rate coefficients is found to be in excellent agreement with the prediction of detailed balance. We also find that the two thermally averaged rate coefficients cross at about $17 \mathrm{~K}$. At lower temperature, $\mathrm{OH}^{-}$shows the larger rate coefficient, whereas at higher temperature the $\mathrm{OD}^{-}$rate becomes larger. This can be explained by the relative importance at different, but fairly close, collision energies of the numerous scattering resonances that appear in the energy-dependent cross-section throughout the range of energies relevant at low temperature. The calculated rates at $22 \mathrm{~K}$ (also plotted in Fig. 3b) agree fairly with the measured rate coefficients at this temperature. The ratio of the two calculated rate coefficients of 0.95 is found to be in quantitative agreement with the experimental ratio. This is a manifestation of the precision that can be achieved in cold collisions when many scattering resonances have to be taken into account. It also confirms the limited predictive power of the simple Langevin model for inelastic ionic collisions at low temperatures.

Selective depletion of the excited rotational state of the hydroxyl anion is not only a means to measure inelastic relaxation rates and compare them with state-to-state $a b$ initio quantum crosssection calculations. It can be used to lower the effective rotational temperature as a prerequisite for further quantum-state-controlled experiments below the temperature of the neutral coolant. We have already achieved an effective rotational temperature for $\mathrm{OD}^{-}$of $16 \pm 1 \mathrm{~K}$ for strong photodetachment laser powers and low buffer gas densities, which corresponds to a depletion of the excited $J=1$ state from $43 \%$ to $32 \%$ (see Supplementary Information). With further development, excited state populations below $1 \%$ would become feasible. More importantly, photodetachment depletion does not rely on specific molecular properties, and is thereby directly applicable to complex polyatomic molecular ions-where, for example, it opens up a powerful approach to prepare internally cold organic molecules of astrophysical relevance, such as polyaromatic hydrocarbons, or biophysical relevance, such as molecular chromophores of fluorescent proteins, for precision 
studies. Understanding inelastic collisions in these systems will provide new insights into the competition of collisional quenching with molecular line emission in radioastronomy and the nonradiative de-excitation of dye molecules.

Received 10 October 2014; accepted 9 April 2015; published online 18 May 2015; corrected after print 1 October 2015

\section{References}

1. Carr, L. D., DeMille, D., Krems, R. V. \& Ye, J. Cold and ultracold molecules: Science, technology and applications. New J. Phys. 11, 055049 (2009).

2. Loh, H. et al. Precision spectroscopy of polarized molecules in an ion trap. Science 342, 1220-1222 (2013).

3. Andre, A. et al. A coherent all-electrical interface between polar molecules and mesoscopic superconducting resonators. Nature Phys. 2, 636-642 (2006).

4. Ratschbacher, L., Zipkes, C., Sias, C. \& Köhl, M. Controlling chemical reactions of a single particle. Nature Phys. 8, 649-652 (2012).

5. Gerlich, D. et al. Ion trap studies of $\mathrm{H}^{-}+\mathrm{H} \rightarrow \mathrm{H}_{2}+\mathrm{e}^{-}$between 10 and $135 \mathrm{~K}$. Astrophys. J. 749, 22-27 (2012).

6. Gerlich, D. et al. Ion-neutral collisions in a 22-pole trap at very low energies. Phys. Scr. T59, 256-263 (1995).

7. Hall, F. H. J. \& Willitsch, S. Millikelvin reactive collisions between sympathetically cooled molecular ions and laser-cooled atoms in an ion-atom hybrid trap. Phys. Rev. Lett. 109, 233202 (2012).

8. Hansen, A. K. et al. Efficient rotational cooling of Coulomb-crystallized molecular ions by a helium buffer gas. Nature 508, 76-79 (2014).

9. Cote, R. \& Dalgarno, A. Ultracold atom-ion collisions. Phys. Rev. A 62, 012709 (2000).

10. Bodo, E. \& Gianturco, F. A. Collisional quenching of molecular rovibrational energies by He buffer loading at ultralow temperatures. Int. Rev. Phys. Chem. 25, 313-351 (2006)

11. Krems, R. V. Cold controlled chemistry. Phys. Chem. Chem. Phys. 10, 4079-4092 (2008).

12. Quéméner, G. \& Julienne, P. S. Ultracold molecules under control! Chem. Rev 112, 4949-5011 (2012).

13. Gilijamse, J. J., Hoekstra, S., van de Meerakker, S. Y. T., Groenenboom, G. C. \& Meijer, G. Near-threshold inelastic collisions using molecular beams with a tunable velocity. Science 313, 1617-1620 (2006).

14. Kirste, M. et al. Quantum-state resolved bimolecular collisions of velocity-controlled OH with NO radicals. Science 338, 1060-1063 (2012).

15. Chefdeville, S. et al. Observation of partial wave resonances in low-energy $\mathrm{O}_{2}-\mathrm{H}_{2}$ inelastic collisions. Science 341, 1094-1096 (2013).

16. Lavert-Ofir, E. et al. Observation of the isotope effect in sub-kelvin reactions. Nature Chem. 6, 332-335 (2014).

17. Tizniti, M. et al. The rate of the $\mathrm{F}+\mathrm{H}_{2}$ reaction at very low temperatures. Nature Chem. 6, 141-145 (2014).

18. Staanum, P. F., Højbjerre, K., Skyt, P. S., Hansen, A. K. \& Drewsen, M. Rotational laser cooling of vibrationally and translationally cold molecular ions. Nature Phys. 6, 271-274 (2010).
19. Schneider, T., Roth, B., Duncker, H., Ernsting, I. \& Schiller, S. All-optical preparation of molecular ions in the rovibrational ground state. Nature Phys. 6, 275-278 (2010).

20. Rellergert, W. G. et al. Evidence for sympathetic vibrational cooling of translationally cold molecules. Nature 495, 490-494 (2013).

21. Schlemmer, S., Kuhn, T., Lescop, E. \& Gerlich, D. Laser excited $\mathrm{N}_{2}{ }^{+}$in a 22-pole ion trap: Experimental studies of rotational relaxation processes. Int. J. Mass Spectrom. 185-187, 589-602 (1999).

22. Schlemmer, S., Lescop, E., von Richthofen, J., Gerlich, D. \& Smith, M. A. Laser induced reactions in a 22-pole ion trap: $\mathrm{C}_{2} \mathrm{H}_{2}{ }^{+}+\mathrm{h} v_{3}+\mathrm{H}_{2} \rightarrow \mathrm{C}_{2} \mathrm{H}_{3}{ }^{+}+\mathrm{H}$. J. Chem. Phys. 117, 2068-2075 (2002).

23. Gerlich, D. et al. State specific stabilization of $\mathrm{H}^{+}+\mathrm{H}_{2}(\mathrm{j})$ collision complexes. J. Phys. Chem. A 117, 10068-10075 (2013).

24. Mikosch, J., Weidemüller, M. \& Wester, R. On the dynamics of chemical reactions of negative ions. Int. Rev. Phys. Chem. 29, 589-617 (2010).

25. Jusko, P., Asvany, O., Wallerstein, A-C., Brünken, S. \& Schlemmer, S. Two-photon rotational action spectroscopy of cold $\mathrm{OH}^{-}$at $1 \mathrm{ppb}$ accuracy. Phys. Rev. Lett. 112, 253005 (2014).

26. Gonzalez-Sanchez, L., Marinetti, F., Bodo, E. \& Gianturco, F. A. OH ${ }^{-}\left(\mathrm{X}^{1} \Sigma^{+}\right)$ collisions with ${ }^{4} \mathrm{He}\left({ }^{1} \mathrm{~S}\right)$ at vanishing energies: A quantum analysis of rotational quenching efficiency. J. Phys. B 39, 1203-1213 (2006).

27. Trippel, S. et al. Photodetachment of cold $\mathrm{OH}^{-}$in a multipole ion trap. Phys. Rev. Lett. 97, 193003 (2006).

28. Otto, R., von Zastrow, A., Best, T. \& Wester, R. Internal state thermometry of cold trapped molecular anions. Phys. Chem. Chem. Phys. 15, 612-618 (2013).

29. Lopez-Duran, D., Bodo, E. \& Gianturco, F. A. Aspin: An all spin scattering code for atom-molecule rovibrationally inelastic cross sections. Comp. Phys. Commun. 179, 821-838 (2008).

\section{Acknowledgements}

This work has been supported by the European Research Council under ERC grant agreement No. 279898. E.S.E. acknowledges support from the Fond National de la

Recherche Luxembourg. We also acknowledge the High-Performance Computing Centre at the University of Innsbruck.

\section{Author contributions}

D.H., S.L., O.L., S.S., E.S.E. and S.S.K. performed the experiments. F.C. and F.G. carried out the calculations. R.W. planned and supervised the project. D.H., F.C., S.S.K. and R.W. prepared the manuscript with input from all authors.

\section{Additional information}

Supplementary information is available in the online version of the paper. Reprints and permissions information is available online at www.nature.com/reprints.

Correspondence and requests for materials should be addressed to R.W.

\section{Competing financial interests}

The authors declare no competing financial interests. 


\section{Corrigendum: Rotational state-changing cold collisions of hydroxyl ions with helium}

Daniel Hauser, Seunghyun Lee, Fabio Carelli, Steen Spieler, Olga Lakhmanskaya, Eric S. Endres, Sunil S. Kumar, Franco Gianturco and Roland Wester

Nature Physics 11, 467-470 (2015); published online 18 May 2015; corrected after print 1 October 2015.

In the version of this Letter originally published the given rotational temperature of the trapped anions was obtained with an incorrect fit function, which also resulted in an overestimation of the measured inelastic rate coefficients. The correct rotational temperature is $22 \mathrm{~K}$. With this rotational temperature the fit to the depletion data yields smaller inelastic collision rates, which are shown in Fig. 2c, and smaller measured inelastic collision rate coefficients, which are shown in Fig. $3 \mathrm{~b}$. Corrected versions of Figs 1, 2c and $3 \mathrm{~b}$ are shown below. The measured rate coefficients are slightly smaller than the computed rate coefficients. Quantitative agreement is confirmed for the ratio of the computed and measured rate coefficients for the $\mathrm{OH}^{-}$and $\mathrm{OD}^{-}$isotopes. These errors have been corrected in the online versions of the Letter.

Figure 1

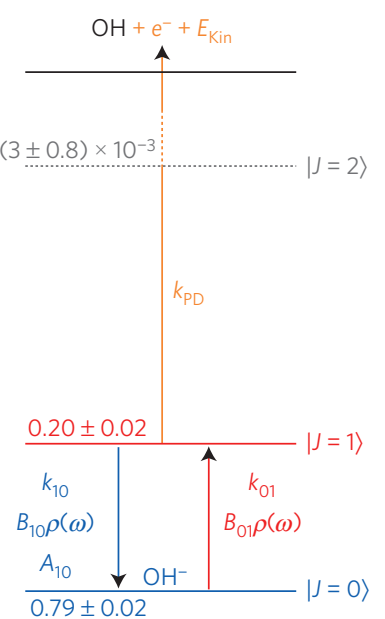

Figure 2c

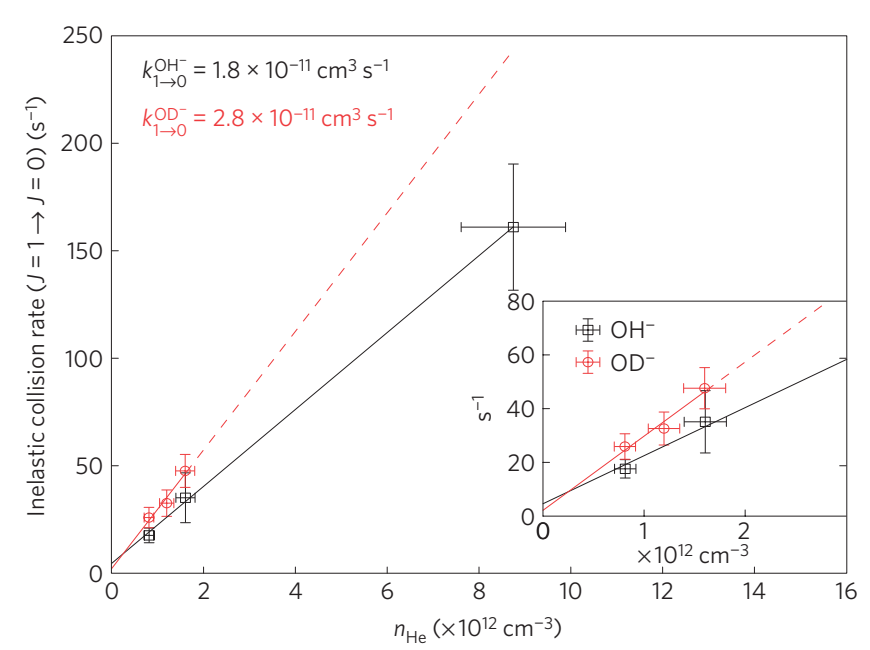

Figure $3 b$

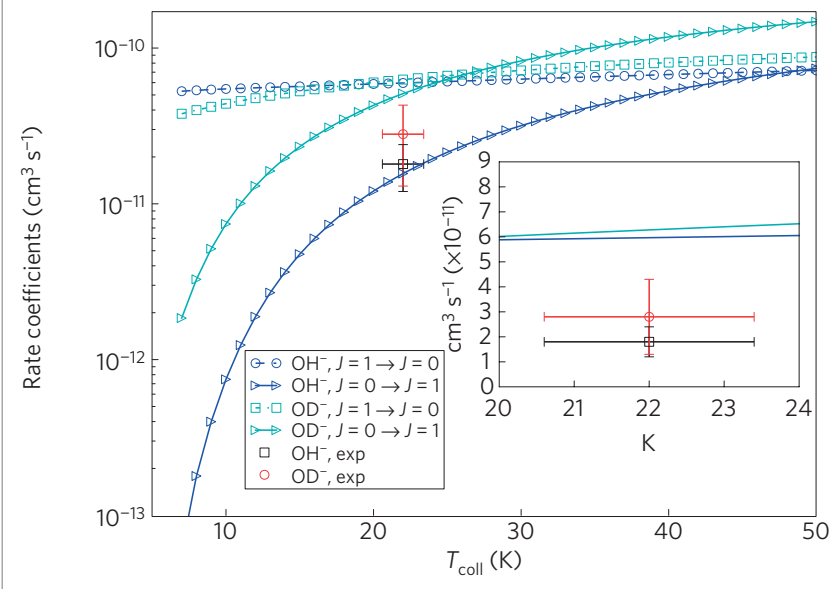

